\title{
Importancia de las probabilidades preprueba en el uso de pruebas diagnósticas
}

\author{
Patricio Herrera L. '; Gastón Duffau T. ${ }^{\text {; }}$ Rosanna Lagos Z. ${ }^{2}$
}

\begin{abstract}
Resumen
Las pruebos diagnósticas son una herramien'a habirual para romar decisiones clinicas, a menudo sesgodas por faclores, entre los cuales uno es no ajusiar los indices variables publicados según las probabilidades preprueba dei paciente individual. Objetivo: mostrar las variaciones de las probabulidades postprueba y sus implicaciones clinico epidemiclógicas, cuando se consideran las probabilidades prepruebo de infección dei traclo urinerio en niños. Diseño y mélodo: Ën dos grupos de niños bajo sospecha clínica de infección urinaria basada en signos inflamclorios de la vía urinario o fiebre de origen no deterninado después de lc cnamnesis y el examen físico, sumados en una tabla $2 \times$ 2 única, según los resultados de probar el comportamiento del examen macroscópico y de una tira reactiva en muestras de orina fresco, se colcularon lo sensibilidad, especificidad y razones de verosimilitud de la combinación de las pruebas para descortar infección de la vía urinaria. Los tiños fueron luego estretficados por edac y sexo para calcular sus valores predictivos, ventajas [odds] y probabilidades de enfermedad poslorueba según las preva'encias determinadas por estas variables. Resultodos: se registraron moderadas a grandes variacionies de los valores predictivos y probabilidades postprueba asacicdos a la edad y sexo, respectivamenle. Las razones de verosimilitud, usadas para calcular los probabilidades posipruebo, se comportaron de modo simila: a los valores predictivos. Lá mavor urilidad de las razones de ve'osimiliturt reside en permitit estas ajustes cuando es necesario calculorlos a partir de una tabla no dicolómica $[2 \mathrm{x}$ ). Se subrava la imporlancia del conocirriento de las probabílidades preprueba para el uso correclo de pruebas diagnósticas.
\end{abstract}

(Palabras clave: pruebas diagnósticas, razones de verosimilituc.í

\section{Diagnostic tests: importance of pre-test probabilities}

Diognostic tests for clinicol purposes are ofien biased by factors like foilure to adjust known variable indexes to individual pretest probabilities. Objective: Io show voriations in postest probabilities, and their clinical and epidemiological implications, after introducing pre-test probabilities of urinary tract infectior in children. Patsents and methods. Two groups of children clinicaly suspected of urinary fract infection by usinory tract inflammotory signs or ever of unrecognizoble origin ofier hislory and physicol, were selected and then summed up into o single two by twc toble, after testing results of maked eye examination of fresh urine specimens plus those with a reaclive strip resl. The combined resis sensitivity, specificity and likelihood ratios for urinory tract infection were recorded. Children were stralified by age and gender-specific predictive values and postlest odds and probabilities of disease. Resul's: wide variations of predictive volues and posttesl probabilities of urinary ircel infection astociated to age and gender groups respeclively were recorded. Likelihood ratios behaved in a similar way than predictive values their main advantage being to allow inclusion of post-tesl probabilities of disease when they cannot be estimated from not dicholomous tobles $(2 \times n)$. The imporlance of pretest probobilities for proper use of dognostic lesls is stressed.

(Key words: diognostic rests, liokility rotios.!

1. Universidad de Chile, Facultad de Medicina, Centro de Epidemiología Clínica (INCLEN). Departamento de Pediatría, Campus Norte.

2. Servicio de Salud Metropolitano Norte, Hospital Roberto del Rio.

Proyecto Fondecyt No 1940049.
El uso y ensayo de pruebas diagnósticas como instrumento del trabajo clínico común y especializado requieren detenida atención por la importancia del papel que desempeñan en las decisiones tomadas sobre los pacientes y por el riesgo de distorsion de tales decisiones, debido 
a los numerosos sesgos potenciales que las afec$\tan ^{1-9}$.

Uno de los sesgos comunes en la interpretación del comportamiento de las pruebas diagnósticas es su aplicación, con criterio intuitivo, basada sclo en la sensibilidad y especificidad declaradas por fos autores que proponen las pruebas diagnósticas, sin reparar que tales índices son, a menudo, poco contiables ${ }^{10}$. O bien, también sólo sobre bases intuitivas, en consideración sólo de los valores predictivos incluidos en la publicación respectiva, calculados a partir de la muestra en que se ensayó la prueba.

El ejercicio que corresponde al clínico, una vez examinada la validez de los resultados derivados del ensayo de una prucba diagnóstica, es analizar la utilidad que tiene ésta en la población en la que se desempeña clínicamente ${ }^{11,1}$. Para ello, es nocesario ajustar los valores predictivos según la prevalencia de la enfermedad en la población que atiende."

El objetivo principal del presente estudio es ilustrar la importancia de las variaciones de la prevalencia, como parte de los atributos del paciente individual ("probabilidad preprueba"), sobrc los índices variables de una prueba diagnóstica. así como sus implicaciones clínicoepidemiologicas, utilizando los resultados de una experiencia real como modelo. Secundariamente, examinar otra manera de usar el comportamiento de una prueba diagnóstica, con otros índices de connotación predictora menos conocidos entre nosotros: Ja razón de verosimilitud ("likelihood ratio" o "LR").

\section{Pacientes y Métodos}

La prueba diagnóstica y los pacientes malizados son parte de una serie de niños con infección del tracto urinario (infección urinaria) comprohada por urocultivos y sedimentos de orina según criterios especificados ${ }^{1 ; .}$ lá, ya analizados con otros fines 15 , its.

De dicha serie se eligieron los niños que fueron referidos a una clínica especializada por padecer de signos de inflamación de las vías urinarias (grupo A), fiebre sin caldsa demostrable luego de la anannesis y examen fisica (grupo B) o signos indirectos de infeccion del tracto urinario (grupo $C$ ). A todos estos niños se les examino la orina mediante una tica reactiva y por examen macroscópico direcıo de la orina recién emitida ${ }^{1+}$. Como la sensibilidad y especificidad del examen directo de orina y tira reactiva difieren entre los grupos, se hizo un examen de comparabilidad estadistica por medio de intervalos de confianza de 95\%, comparación de proporciones con prueba de $Z$ y aná- lisis de heterogeneidad con la prueba de Mantel Haens$z^{17}$. Luego de este análisis se construyó una tabla de $2 \times 2$ a partir de la suma de los grupos A y B y se calcularon los valores de sensibilidad, especificidad $y$ razones de verosimilitud. según las técnicas habituales para los resultados positivos y negativos de la prueba ${ }^{3}$. La prevalencia se analizó según los grupos de edad, sexo y la variante clínica representada por el grupo C. a partir de valores obtenidos de uno de los estudios preliminares practicados en estos niōos 1?, según ta fórmula: n de infecciones urina. rias confinnadas / $\mathrm{n}$ de pacientes referidos por sospecharse infección urinaria, en cada segmento de edad, sexo y de la variante clínica (grupo C)

\section{Resultados}

Se incluyeron en el estudio 1204 niños, 1005 corresponden a la suma de los grupos A y $B(A B)$ y 199 al grupo $C^{14}$. Las prevalencias por edad y sexo del grupo AB aparecen en la tabla 1. Estos valores son considerados en este análisis como probabilidades preprueba. Las tablas correspondientes a las distribuciones determinadas por el comportamiento de la prueba en estudio en los grupos de niños con signos inflamatorios de las vías urinarias (grupo A) y con fiebre de origen no precisado por la amamnesis y examen físico (grupo B), aparecen en la tabla 2, en la que se incluyen sus índices fijos, además del resultado de las pruebas usadas en examinar su homogeneidad. Ambas tablas fueron combinables porque la prueba de heterogeneidad, así como los intervalos de confianza de $95 \%$ y la prueba de $\mathbf{Z}$ impiden considerarlas provenientes de universos diferentes, con niveles de probabi-

\section{Tabla 1}

\section{Probabilidades preprueba (prevalencia) según la edad y sexo $^{13}$}

\begin{tabular}{lrrrr}
\hline Grupos por edad: & & & & \\
Edad (meses) & $0-23$ & $24-47$ & $48-71$ & $>71$ \\
n referidos & 196,0 & 251,0 & 168.0 & 390,0 \\
\% confirmados & 34,0 & 49,0 & 60,0 & 63,0 \\
\hline Grupos por sexo: & & & & \\
Sexo & Varones & & Mujeres \\
n referidos & & 221,0 & & 784,0 \\
\% confitmados & 33,0 & & 59,0 \\
\hline
\end{tabular}




\section{Tabla 2}

Tablas de origen y tabla construida en 821 niños referidos por sospecha de infección de la vía urinaria ${ }^{1.3}$

Tabla de origen

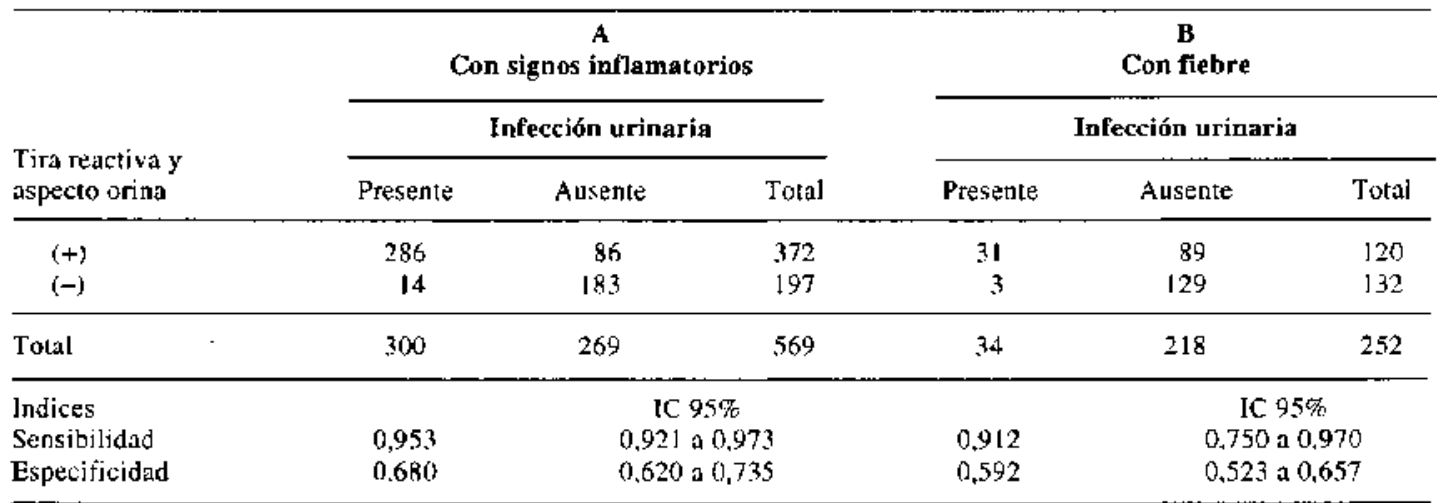

Prueba de heterogeneidad de Mantel-Haenszel: $\chi 2=2.38 ; p>0,10$.

Prueba de $Z$ : Sensibilidad de A vs, sensibilidad de $B: Z=0,625 ; p>0.5$.

Especificidad de A vs. especificidad de B: $Z=1,924 ; \mathrm{p}>0,05$

Tabla de la suma de los nin̄os bajo sospecha $(A+B)$

\begin{tabular}{lrrr}
\hline \multirow{2}{*}{$\begin{array}{l}\text { Tira reactiva } \\
\text { aspecto orina }\end{array}$} & \multicolumn{3}{c}{ Infección urinaria } \\
\cline { 2 - 4 } \multicolumn{1}{c}{$(+)$} & Presente & Ausente & Toral \\
\hline Total & 317 (a) & 175 (b) & 312 (d) \\
\hline Sensibilidad & 37 (c) & 487 & 329 \\
Especificidad & 334 & & 821 \\
LR+: 2,64 & & 0.949 & \\
LR-: 0.08 & 0.640 & & \\
\hline
\end{tabular}

LR+: Razón de verosimilitud pasa prueba positiva: $(a /(a+c) /(b /(b+d)$.

LR-: Razón de verosímilitud para prueba negaliva. $(c /(a+c) /(d / a b+d)$.

lidades $<0,05$. No ocurrió lo mismo con el grupo $\mathrm{C}$, que se reservó para estimar los valores de los índices variables en el grupo con más baja prevalencia. De este modo, la tabla 3 es originada por la combinación de los dos grupos sumados y a partir de ella se calcularon sus índices fijos y sus razones de verosimilitud para resultados positivos y negativos ( $L R+$ y LR-). Los cálculos de las variaciones de probabilidades pre y postprueba están estimados a partir de esta tabla, usando las diferentes prevalencias por edad o sexo, respectivamente. En la tabla 3 se anotan los cálculos de las probabilidades pre y postprueba, examinados según los índices fijos sensibilidad y especificidad y razones de verosimilitud, según las probabilidades preprueba de cada grupo etario. En la tabla 4 se presentan los cálculos según el sexo y se anotan las fórmulas empleadas para los cálculos. En la tabla 5 se presentan lias probabilidades pre y postprueba al aplicarla al grupo con signos indirectos de in. fección urinaria. 


\section{Tabla 3}

Utilización diagnóstica del examen macroscópico de orina y una tira reactiva en niños con síntomas clínicos de infección del tracto utinario:

probabilidades pre y postprueba según prevalencia dependiente de la edad ${ }^{14}$

a) Análisis predictivo basado en sensibilidad y especificidad

Probabilidades preprueba:

\begin{tabular}{lcccc}
\hline Edad (meses) & $\mathbf{0 ~ a ~ 2 3}$ & $\mathbf{2 4 \times 4 7}$ & $\mathbf{4 8 \times 7 1}$ & $>\mathbf{7 1}$ \\
\hline Probabilidad preprueba & $\mathbf{0 , 3 4}$ & $\mathbf{0 , 4 9}$ & $\mathbf{0 , 6 0}$ & $\mathbf{0 , 6 3}$ \\
Sensibilidad (+): $\quad 0,95$ & & & \\
Especificidad (-): 0,64 & & 0,720 & 0,799 & 0,82 \\
Valor predictivo positivo & 0,57 & 0,93 & 0.89 & 0.88 \\
Valor predictivo negativo & 0,96 & & \\
\hline
\end{tabular}

b) Análisis predictivo basado en razón de verosimilitud

\begin{tabular}{|c|c|c|c|c|}
\hline Edad (meses) & ( a 23 & 24 a 47 & $48 \times 71$ & $>71$ \\
\hline Probabilidad preprueba & 0,34 & 0,49 & 0,60 & 0,63 \\
\hline Ventaja preprueba & 0,52 & 0,96 & 1,50 & 1,70 \\
\hline \multicolumn{5}{|l|}{$L R+: 2.64$} \\
\hline Ventaja postprueba & 1.36 & 2.54 & 3.96 & 4,50 \\
\hline \multicolumn{5}{|l|}{ LR-: 0,08} \\
\hline Ventaja pastprueba & 0,04 & 0,08 & 0,12 & 0,14 \\
\hline Probabilidad postprueba $(+)$ : & 0.57 & 0,72 & 0.80 & 0.82 \\
\hline Probabilidad postprueba (-): & 0,04 & 0.07 & 0.11 & 0,12 \\
\hline
\end{tabular}

Ventaja preprueba = probabilidad prepruebal(1-probabilidad preprueba).

Probabilidad postprueba = ventaja postprueba $(\mathbf{I}+$ ventaja postprueba $)$.

Ajuste de probabilidad preprueba usando razón de verosimilitud: ventoja postprueba $=$ ventaja preprueba $\times$ razón de verosimilitud.

LR: razón de verosimilitud para prueba positiva (+) o negatiqa (-). 


\section{Tabla 4}

Utilización diagnóstica del examen macroscópico de orina y una tira reactiva en niños con síntomas clínicos de infección del tracto urinario: probabilidades pre y postprueba según prevalencia dependiente del sexo

a) Análisis predictivo basado en sensibilidad y especificidad

\begin{tabular}{lcc}
\hline Grupo por sexo: & Varones & Mujeres \\
\hline Probabilidad preprueba & $\mathbf{0 , 3 3}$ & $\mathbf{0 , 5 9}$ \\
Sensibilidad (+): 0,95 & & \\
Especificidad(-): 0,64 & & \\
Valor predictivo positivo & 0,57 & 0,791 \\
Valor predictivo negativo & 0,96 & 0,90 \\
\hline
\end{tabular}

b) Análisis predictivo basado en razón de verosimilitud

\begin{tabular}{|c|c|c|}
\hline Grupos por sexo: & Varones & Mujeres \\
\hline Probabilidad preprueba & 0,33 & 0,59 \\
\hline $\begin{array}{l}\text { Ventaja preprueba } \\
\text { LR+: } 2,64\end{array}$ & 0,49 & 1,44 \\
\hline $\begin{array}{l}\text { Ventaja postprueba } \\
\text { LR-: } 0,08\end{array}$ & $1,3^{\circ}$ & 3,8 \\
\hline Ventaja postprueba & 0,04 & 0,12 \\
\hline Ptobabilidad postprueba (t) & 0.57 & 0.79 \\
\hline Probabilidad postprueba $(-)$ & 0.04 & 0,1 \\
\hline
\end{tabular}

LR: razón de verosimulitud para prueba positiva (+) o negativa (-)

\section{Comentario}

Cuando se estudian los principios sobre el comportamiento de una prueba diagnóstica, los ejemplos suelen presentarse de modo que los números de casos del grupo que tiene la enfermedad $(a+c)$ y el grupo de casos que no la tiene $(b+d)$ son iguales, es decir, la prevalencia, en el grupo experimental, es $50 \%$ (1:1). En los grupos de la literatura en que se proponen pruebas diagnósticas es común que, si esta razón no es $1: 1$, de todos modos la prevalencia de la tabla publicada (tabja experimental) resulta cercana a esta proporción. Los valores predictivos (índices variables) calculados en estas condiciones, con alta probabilidad no serán útiles para
Tabla 5

Utilización diagnóstica deI examen macroscópico de orina y una tira reactiva en niños con síntomas clínicos de infección del tracto urinario (probabilidades pre y postprueba de infección urinaria según prevalencia en una categoría de presentación clínica)

\section{a) Análisis predictivo basado en sensibilidad y especificidad}

Probabilidad preprueba $\quad 0,15$

Sensibilidad (+): $\quad 0,95$

Especificidad (-): 0,64

Valor predictivo positivo

0.31

Valor predictivo negativo

0,99

b) And́lists predictivo basado en razón de verosimilitud

\begin{tabular}{lc}
\hline Probabilidad preprueba & $\mathbf{0 , 1 5}$ \\
\hline Odds preprueba & 0,17 \\
LR+: 2,64 & \\
Odds postprueba & 0,45 \\
LR-: 0,08 & \\
Odds postprueba & 0,01 \\
Probabilidad postprueba & 0,31 \\
Probabilidad postprueba & 0,01
\end{tabular}

LR: razón de verosimilited para prueba positiva $\{+\}$ o negativa $\{-\}$

nuestro trabajo clínico, sobre todo si no es muy especializado, ya que éste se lleva a cabo en poblaciones en las que la prevalencia de la enfermedad es más baja, o es variable. Como los índices fijos (sensibilidad, especificidad, razón de verosimilitud) son considerados estables y no dependientes de la prevalencia, nos es posible ajustar los valores predictivos en cada situación. Esta es la razón por la cual los índices variables correspondientes no aparecen en la tabla 2, que debe ser considerada experimental, porque su prevalencia es sólo la "cruda" y sus valores predictivos deben ser ajustados según características de cada paciente, que es el ejercicio que se desarrolla mós adelante. 
El examen de los valores derivados de la ta. bla 2 muestran el típico comportamiento de los indices variables (valores predictivos, probabilidades postprueba) con las variaciones de la prevalencia (tablas 3 y 5). Así es como la prevalencia afecta los valores predictivos $y$, en general, las probabilidades postprueba, de modo que las diferencias de prevalencia tienen efecto sobre los valores predictivos, lanto mayores cuanto mayor sea la magnitud de tales diferencias. Los conceptos analizados y su valor operacional tienen consecuencias importantes en aspectos clínicos y epidemiológicos.

La primera consideración necesaria es recordar que estamos tratando con hechos reales, con un grupo de pacientes que incluye niños con signos inflamatorios de las vías urinarias, fiebre de origen no precisado después de conocer su anamnesis y examen físico o signos indirectos de infección de la vía urinaria. Ello implica tener presente que, usando un modelo real con posibilidades de aplicación directa, derivamos generalizaciones que deben entenderse como hechas a partir de este modelo en particular (v.g., las decisiones clínicas gue aparecen no están, necesariamente. ligadas a hos resultados de! comporlamiento de cualquier otra prueba o cualquier otra enfermedad). Debe advertirse, además, que el análisis que sigue reconoce algunas restricciones inherentes a todo modelo clínjco real ${ }^{18,19}$ pero las ignora, ya que, además de no invalidar necesariamente la utilización de] modelo y las conclusiones principales, la sujeción a la totalidad de las restricciones hipotéticas haría imposible la aplicación clínica de toda prueba diagnóstica.

El ajuste de probabilidades preprueba a las de postprueba, tiene la importancia de dar sentido práclico a las pruebas diagnósticas que tanto apreciamos y usamos los clínicos. En cste contexto, los términos "prevalencia" o "probabilidades preprueba" se refieren a la probabilidad específica de determinado paciente de sufrir la enfermedad que nos interesa, en este caso, infección urinaria. El primer hecho que cabe hacer notar, es que la "prevalencia" $((a+c) /(a+b+c+d))$ en la tabla $2 \times 2$ en que se estudió el comportamiento de la prueba diagnostica (tabla 2) es $40.7 \%$, que no representa, ni la prevalencia de la intección en niños del área norte de Santiago, ni la probabilidad preprueba de cualquier niño de esta población tomado al azar, sino sólo un va- lor resultante de una situación experimental. La probabilidad individual, diferente de la probabilidad de la población general (v.gr., de niños del área norte de Santiago) y de la situación experimental, está determinada de modo empírico, en el modelo analizado, por hechos de la anamnesis y examen físico, tales como la edad, el sexo y una cierta categoría clínica que hace suponer infección del tracto urinario. Por ejemplo, ел un niño del grupo "0 a 23 meses" -cuya probabilidad preprueba es 0,34 - el valor predictivo positivo de padecer la enfermedad es 0,57 . Es decir, en este caso, la prueba es poco informativa o aclaradora porque la probabilidad está cercana a $50: 50 \%$. La probabilidad postprueba -usando las razones de verosimilitud- le asigna, a su vez, una probabilidad semejante, lo que inplica que la de infección uriunaria, para este niño. sigue estando cerca de $50 \%$ y que la probabilidad postprueba equivale al valor predictivo negativo. En cambio, para un nifno del grupo "mayor de 71 meses", cuya probabilidad preprueba es 0,63 , el valor predictivo positivo y la probabilidad postprueba llegan a 0,82. En este caso, la probabilidad de infección urinaria es aproximadamente 4 a 1 . De modo semejante, en un niño del grupo "0 a 23 meses", con un resultado negativo de esta prueba, dada su probabilidad preprueba, el valor predictivo negativo es 0,96 , lo que implica que la probabilidad de esa infección es casi nula (con error de $4 \%$ ). En el niño del grupo "mayor de 71 meses", en cambio, el valor predictivo negativo es 0,88 y la probabilidad postprueba negativa, 0,12. Este resultado, que puede parecer razonablemente suficiente para descartar infección de la vía urinaria, pucde no serlo dependiendo de la naturaleza de la enfermedad en estudio, es decir, a la vista de las consecuencias de aceptarlo para excluir la enfermedad o para no considerarlo suficiente para ello (v.gr., tratarlo o no tratato, proseguir el estudio o no, etc.).

Si la edad representa un aspecto del paciente individual que requiere ajustar los valores predictivos de esta prucba diagnóstica, el ajuste por sexo resulta más ilustrativo aun de la importancia de hacerlo. Un varón con el síndrome descrito, con su probabilidad preprueba 0,33, el resultado positivo le asigna la probabilidad 0,565 $(56,5 \%)$ de tener, efectivamente infección urinaria, lo cual no resulta del todo concluyente. En cambio, el resultado negativo implica valor pre- 
dictivo negativo $=0,963$, es decir, riesgo de la infección $=0,037$ o $3,7 \%(1-0,963)$, lo que sí es ilustrativo: muy probablemente, el clínico no enviará este niño a haçerse más exámenes ni le iniciará (es de esperar) tratamiento. En el caso de una mujer, el problema es diferente porque sus probabilidades preprueba son altas (mayores que el $50 \%$, de modo que un examen positivo tendrá valor predictivo positivo de casi $80 \%$ (4: 1 a favor de infección), por lo que probablemente se iniciará el tratamiento y se la referirá para el resto de los exámenes necesarios. Un resultado negativo, en esta mujer, dejará un margen de $10 \%$ de error por presencia de la enfermedad (valor predictivo negativo $=0,90$ ). La decisión, en este caso, dependerá del clínico y de posibles otros antecedentes a la mano. Pero, en forma quizás diferente de la situación de un niño con probable ITU, $10 \%$ de duda es demasiado si la enfermedad amenaza la vida del paciente, de modo que deba ignorarse este margen de error y se prosiga el estudio y se haga terapia, como si sólo se pudiera confiar en valores predictivos negativos $=1(100 \%)$ lo que, como se puede observar, esta prueba para ITU no proporciona. En el caso del grupo con signos indirectos de ITU puede apreciarse, del mismo modo, que los valores predictivos negativos tienen valores predictivos más extremos. debido a la baja prevalencia de infección urinaria en el grupo (vator de la probabilidad preprueba). Debe señalarse, sin embargo, que esta última estimación se incluye sólo para ilustrar las variaciones de los índices variables según la prevalencia pero, en este caso, el ajuste por edad o sexo sería más inexacto que los anteriores, debido a la reducción del número de casos al estratificar el grupo por estas variables.

Siguiendo con el modelo en estudio se puede comprender el alcance de la extensión epidemiologica de este análisis. En los grupos de baja prevalencia de infección urinaria, por ejemplo 0,34 (ya definidas las condiciones clínicas y en el grupo de 0 a 23 meses), la aplicación de esta prueba permite descartar la enfermedad en el $96,1 \%$ de los casos cuando su resultado es negativo. En otras palabras, de resultados negativos en 100 niños con probabilidad preprueba 0,34 se puede evitar el envío de estos pacientes al hospital (por ejemplo, para sedimento urinario, cultivo, antibiograma, recuento de colonias y otras consecuencias fáciles de prever del diag- nóstico de la afección), con probabilidad de error de no haber enviado s6lo a 4 niños con la enfermedad verdadera (obviamente. todos estos niños deben ser objeto de vigilancia clínica por su médico, en su consultorio). Ello podría evitar un exceso de tratamientos antibíticos, de exámenes de laboratorio más complejos, de ansiedad de los padres, entre otras.

Las razones de verosimilitud (LR) expresan cuanto más probable es que cierto resultado positivo o negativo provenga de una persona con $(L R+)$ o $\sin (L R-)$ la enfermedad. Su desarrollo y cálculo resultan algo más engorrosos que el de los valores predictivos, porque en ello están involucrados dos conceptos poco familiares: la conversión de "probabilidades simples" (proporciones o porcentajes) a ventaja ("odds") y viceversa, así como el concepto de "verosimilitud", que no es sino otra manera de expresar probabilidades. En suma, el proceso consiste en lo siguiente: a) transformación de probabilidad en ventaja (ventaja preprueba), b) corrección de éste (multiplicación) por la razón de verosimilitud (LR) correspondiente, y c) conversión del resultado (en ventaja) a probabilidades simples (proporción o porcentaje) ${ }^{3}$. Hay autores que están asignando mayor importancia al uso de LR que a los índices calculados a partir de la sensibilidad y especificidad, aduciendo varias ventajas de los primeros sobre los segundos ${ }^{2}, 11,12$, lo cual podría o no ser aceptado por nosotros. Sin embargo, una clara ventaja de las razones de verosimilitud es la posibilidad de calcular las probabilidades postprueba a partir de pruebas diagnósticas que no entregan resultados dicotómicos, es decir, que en vez de tablas $2 \times 2$, usan tablas $2 \times \mathbf{n}$, como podrían ser las categorías de probabilidad de cierta enfermedad. Cuando se usa en esta circunstancia, la solución por medio de razones de verosimilitud permite, además, conseryar valiosa información diagnóstica si se la compara con la solución consistente en reducir tablas $2 x$ n a dicotómicas, variando el "punto de corte" en la escala de categorías ${ }^{20,21}$.

Sin embargo, el uso de valores predictivos parece tener ventaja sobre el uso de predicciones diagnosticas basadas en razones de verosimilitud, pero ello es sólo aparente si se considera que los cálculos se hacen en tablas de carácter experimental. Al momento de ajustar las probabilidades preprueba y conociendo s6 6 la sensibilidad y especificidad de cierta prueba 
para obtener las probabilidades postprueba en un caso individual, los procedimientos aritméticos pueden ser igualmente engorrosos para, finalmente y expresando las ventajas postprueba en probabilidades simples, tener resultados equivalentes a los de los valores predictivos.

El examen de este modelo con los fines anotados no puede terminar sin algunos alcances necesarios. Primero, es que el uso del término "prevalencia" se justifica porque se refiere a la proporción en que cierto fenómeno (enfermedad) está presente en una población definida. Es necesaria esta explicación, porque el uso general de "prevalencia" puede sugerir la referente a la existencia de casos de una enfermedad en determinada población general que, en este caso, está representada por la tasa de casos de infección urinaria incidentes en niños de la población adscrita al Servicio de Salud Metropolitano Norte, a través del estudio referido y que fue $3,7 / 1000<15$ años $^{13}$. Esta aclaración es importante porque el clínico que usará pruebas diagnósticas no debe buscar el dato "probabilidad preprueba" en estudios de prevalencia o incidencia poblacionales de ciertas enfermedades sino, más bien, en estudios clínicos epidemiológicos especialmente dirigidos a reconocer riesgos diferenciales en grupos de pacientes definidos clínicamente como presuntamente afectados por la enfermedad que interesa, y para la cual se ha propuesto una determinada prueba (v.gr., la examinada como modelo en este análjsis).

Si bien la prueba aumentó la exactitud del diagnóstico clínico según los grupos etarios y e] sexo, es posible que el mayor aporte de ella lo constituya la baja probabilidad de la enfermedad cuando su resultado es negativo, ya que el valor predictivo negativo alcanza, sobre todo en los grupos de menor prevalencia, a más de $96 \%$, lo que implica que el riesgo de exisir la enfermedad es $<4 \%(1-0,96)$. Este aspecto hace de su validación un conveniente objetivo en el futuro.

Es fácil deducir de este ejercicio que, a mayor información cuantitativa sobre las características de deterninado paciente (valor de probabilidad preprueba determinado por diversas variables, por ejemplo, biográficas), mayor la exactitud que se obtendrá al aplicar determinada prueba diagnóstica. En general, en pediatría, pese a la gran cantidad de trabajos descriptivos presentados a congresos o publicados, no es fácil encontrar aquellos que ofrezcan estimaciones válidas de incidencias o prevalencias según variables de interés en relación a la aplicación e interpretación de pruebas diagnósticas, elemento indispensable para el correcto uso de éstas.

\section{Agradecimientos}

Al Dc.Carlos Valenzuela Y., por la revisión del origimal y sus valiosas sugerencias.

\section{Referencias}

1. Ransohouff DF. Feinslein AR: Problems of spectrum and bias in evaluating the efficay of diagnostic tests. NEJM 1978: 299: 926-930.

2. Riegelman RK: Studying a study and testing a rest. Little, Brown and Co. Boston $198 \mathrm{I}$.

3. Sackett $D L$, Haynes RB, Tugwell P: Clnical Epidemiology. Litte Brown and Co. Boston 1985.

4 Feinstein $A R$ : On the sensitivity, specificity, and discrimination of diagnostic tests. Clin Pharmacol Therap 1975; 17; 104-116.

5. Harris MM: The hazards of bedside Bayes. JAMA 1981; 246: 2602-2605.

6. Vulenstein PN: Evaluating diagnostic tests with imperfect standards. Am J Clin Pathol 1990; 93: 252258.

7. Sacketr DL, Haynes RB, Tugwell P: Clinical epidemiology Little, Brown \& Co, Boston 1985.

8. Weinstein $M C$, Fineberg $H V$ - Clinical decision analysis, W.B. Saunders Co. Philadelphia, 1980.

9. Herrera P, Lagos $R$ : Contribución al estudio de pruebas diagnósticas: Sobre sus indices fijos y valores predictivos. Rev Chil Pediatr 1995; 66: 209-216.

10. Herjera P. Duffau $G$ : Pruebas diagnósticas revisión de algunos aspectos cuantitativos para su desarollo e interpretación. Rev Chil Pediatr 1995: 66: 335-340.

11. Jaeschke R. Guyatt $G H$, Sackett DL: User's guide to the medical literature. How to use an article about a diagnoslic test. A. Are the results valid?. JAMA 1994; 227।: 389-391.

12. Jaesche R, Glyan GR, Sackets DL: User's guide to the medical literature. III. How to use an article about a diagnostic test. $B$. What are the results and will they help me in caring my patients?. JAMA 1994: 271: 703-707.

13. Lagos $R$. Carter J, Benavente $C$ et ar: Epidemiología de las infecciones del tracto urinario en niños y riesgo de recurrencia $y$ alteraciones nefrourológicas. Rev Chil Pedintr 1995; 66: 1-12.

14. Lagos $R$, Carter J, Herrera P: Utilidad de una tira reactiva y del aspecto macroscópico de la orina para descartar la sospecha clínica de infección del tracto urinario en niffos ambulatorios. Rev Chil Pediatr 1994; 65: $88-94$.

15. Herrera P. Lagor R. Muñoz. A, et al: Asociación de matcadores etitrocitarios (ABO, MNSs, tH, Lewis, P1) 
con recurrencias y anomalías matómicas y funcionales en niōos con infección del tracto urinario Rey Chil Pediatr 1996; 67: 109-111.

16. Lagos $R$, Herrera $P$. Seputveda $A$, et ol: Pactores de urovirulencia bactetianos: Asociación con recurrencias y anomalías anatónicas y funcionales en niños con infeccion del tracto urinario. Rev Med Chil 1966: 124: 1211-1218.

17. Schlesselman JJ: Case-control scudies. Oxford University Press, NY, 1982.

18. Hetrera $P$, Lagos $R$ : Contribución al estudio de pruebas diagnósticas: Sobre sus indices fijos y valores predictivos. Rev Chil Pediatr 1995; 66: 209-2 I6.
19. Herrera P, Duffau G: Pruebas diagnósticas: Revisión de algunos aspectos cuantitativos para su desarrollo e interpretación. Rev Chil Pediatr 1995; 66: 335-340.

20. Jaeschke $R$. Guyatt $G$. Sackett $D L$ : User's guides to the medical literature. IU. How to use an article about a diagnostic test. A. Are the results valid?. JAMA 1994; 27 l: 389-39].

21. Jaeschke $R$. Guyatt $G$, Sackett $D L$ : User's guides to the medical literature. III. How to use an article about a diagnostic test. B. What are the results and will they help me in caring for my patients?. JAMA 1994; 271 : 703-707.

\section{AVISO A LOS AUTORES}

Con el objeto de dar prioridad a los trabajos de investigación, en vista de las limitaciones de espacio de la Revista Chilena de Pediatría, el Comité Editorial ha acordado restringir la impresión de casos clínicos a un máximo de dos por cada número. 